\title{
Composition minérale et organique de différents lactosérums acides industriels, analysée par électrophorèse capillaire
}

\author{
F Saulnier, M Calco, G Humbert, G Linden \\ Laboratoire des biosciences de l'aliment, unité associée à l'Inra, université Henri-Poincaré, \\ Nancy-I, BP 239, 54506 Vandœuvre-lès-Nancy cedex, France
}

(Reçu le 22 juin 1995 ; accepté le 6 mai 1996)

\begin{abstract}
Résumé - Compte tenu de leur importante minéralisation, les lactosérums acides industriels sont plus difficilement valorisables que les lactosérums doux. Aussi, avant de choisir un traitement de valorisation, il est essentiel de connaître rapidement leurs compositions organo-minérales. Nous avons utilisé, pour cette étude, l'électrophorèse capillaire et déterminé la composition minérale et organique de 12 lactosérums acides industriels d'égouttage, issus de diverses technologies fromagères (pâtes molles $A O C$ et pâtes fraîches). Une grande hétérogénéité de la fraction minérale et organique a été mise en évidence. Des différences significatives entre ces deux technologies ont été observées pour les teneurs en potassium, calcium, magnésium, phosphate, acétate et lactate. De fortes corrélations entre phosphate, calcium et lactate sont à relier avec l'origine technologique des lactosérums, à savoir les protocoles de fabrications fromagères dont ils sont issus. Une corrélation hautement significative existe entre le taux d'acétate et celui du lactate pour les lactosérums acides issus de technologies pâtes molles.
\end{abstract}

électrophorèse capillaire / lactosérum acide / minéraux / acide organique

Summary - Organic and mineral composition from industrial acid wheys analysed by capillary electrophoresis. Valorization of industrial acid wheys is one of the greatest problem of the dairy industry. In fact, their mineralization is superior to that of sweet wheys. Before considering new utilizations, it is necessary to determine rapidly their organic and mineral compositions. The mineral and organic composition of 12 industrial acid wheys from different cheese making (AOC-moulded curd and fresh curd) were studied by capillary electrophoresis. An important heterogeneity of the mineral and organic phases is shown. There are significant differences between these two technologies, particulary in the rate of calcium, potassium, magnesium, phosphate, acetate and lactate. There are significant correlations between phosphate, calcium, lactate which could be explained by cheese making. We found a significant correlation between acetate and lactate for acid wheys from AOCmoulded curd technology.

capillary electrophoresis / acid whey / mineral / organic acid 


\section{INTRODUCTION}

La fabrication des fromages et l'extraction de la caséine du lait écrémé laissent comme produit dérivé un liquide clair, jaune verdâtre : le lactosérum (Rolet, 1920) qui a une composition variable avec le type de fabrication dont il provient. On distingue le lactosérum " doux " et le lactosérum " acide " selon que son acidité est respectivement inférieure ou supérieure à $18^{\circ}$ Dornic (Meréo, 1971).

De ces deux types de lactosérum, le lactosérum doux est le plus facilement valorisé sur le plan industriel. II contient environ 63 à $67 \mathrm{~g}$ de matière sèche (MS) par litre, dont 45 à $50 \mathrm{~g}$ de lactose, 7 à $9 \mathrm{~g}$ de matières azotées, 6 à $8 \mathrm{~g}$ de matières minérales, 1 à $2 \mathrm{~g}$ de matière grasse (Blanc, 1969 ; Veisseyre, 1979 ; Alais, 1984). Les lactosérums acides ont une composition plus variable que les lactosérums doux (Veisseyre, 1979 ; Saulnier et al, 1995). De plus, ils renferment moins de lactose et plus de sels minéraux que les lactosérums doux (Webb, 1972 ; Hargrove et al, 1976).

Par sa faible teneur en lactose et protéines, et sa forte minéralisation, le lactosérum acide est moins bien valorisé (Alais, 1981). De plus, selon Alais (1984), son séchage est délicat par risque de colmatage ou de mottage.

A la fin des années 1980, une nouvelle technique de dosage des minéraux et acides organiques est apparue sur le marché : la CIA ${ }^{\mathrm{TM}}$ (capillary ion analysis) (Waters, Milford, MA, États-Unis). La CIA ${ }^{T M}$ est une technique optimisée pour l'analyse des ions minéraux et organiques de faible taille et fortement mobiles. Ses avantages résident dans la rapidité d'analyse, la facilité d'utilisation face à la chromatographie ionique mais surtout par l'absence de traitement de l'échantillon et le faible coût d'analyse (Saulnier et al, 1992 ; Schmitt et al, 1993).

Ainsi, compte tenu de la grande hétérogénéité de la composition minérale et orga- nique des lactosérums acides, et avant d'envisager un type de valorisation (Kelly, 1991 ; Pontalier et al, 1995), il est souhaitable de connaître rapidement leurs compositions à l'aide d'une méthode qui est en train de prendre une place importante parmi les méthodes analytiques de biochimie. Selon Lindeberg (1995), 80 applications de l'électrophorèse capillaire ont été recensées en agro-alimentaire dont plus de $50 \%$ pour les ions jusqu'à présent.

\section{MATÉRIELS ET MÉTHODES}

\section{Échantillons de lactosérums}

Les lactosérums acides industriels (extrait sec (ES) et pH moyens respectivement de $6 \%$ et 4,60 ) sont des lactosérums d'égouttage de différentes technologies fromagères :

- cinq sont issus d'une technologie pâtes fraîches (coagulation lente après acidification par des bactéries lactiques du lait et séparation du caillé-lactosérum par centrifugation) de cinq usines différentes ;

- sept autres sont issus de technologies pâtes molles AOC de sept usines différentes.

Tous ces lactoserums ont été collectés par la SA Lacto Sérum France (Verdun, France). La fraction aliquote est prélevée au sein de mélange de 20000 à 100000 litres de lactosérum. Après échantillonnage, ils sont congelés a $-20^{\circ} \mathrm{C}$. Deux lactosérums de technologies pâtes fraîches n'ont pas pu être analysés à $6 \%$ d'ES car ils ont été préconcentrés par osmose inverse (Paterson Candy International, Hampshire, Grande-Bretagne) à 20,5\% d'ES.

\section{Dilution des échantillons}

Les lactosérums après décongélation (15 heures à $4{ }^{\circ} \mathrm{C}$ ) et agitation, sont dilués au 1/200 avec de l'eau Ultra Pure (Millipore $\mathrm{CO}$, Bedford, MA, États-Unis).

\section{Standards utilisés}

Ils proviennent de chez Analys SA (Manosque, France), Kalokit 1 pour les anions et Kalokit 2 pour les cations. Une courbe d'étalonnage est réalisée avec cinq concentrations différentes de standards. 


\section{Matériel}

L'appareil d'électrophorèse capillaire est le modèle Quanta 4000 (Waters, Milford, MA, ÉtatsUnis). Le capillaire utilisé est en silice fondue, d'un diamètre interne de $75 \mu \mathrm{m}$ et d'une longueur de $60 \mathrm{~cm}$. L'acquisition et l'exploitation des résultats sont réalisées à l'aide du logiciel Maxima 820 (Waters, Milford, MA, États-Unis).

\section{Dosage des cations}

La détermination des cations a été optimisée et validée par Bondoux et al (1991), Saulnier et al (1992) et Schmitt et al (1993). L'électrolyte est composé de Tropolone $(-2,4,6$-cycloheptatriènenone), de 18 -crown- $6(1,4,7,10,13,16$-hexa oxacyclo-octadécane) (Sigma, Saint-Louis, États-Unis) et de UV CAT 2 (Waters, Milford, MA, États-Unis). L'injection est réalisée en mode hydrostatique durant $30 \mathrm{~s}$ (volume injecté de $10 \mathrm{~nL}$ ). La détection se fait à $185 \mathrm{~nm}$ (fig 1).

\section{Dosage des anions}

La détermination des anions a été optimisée et validée par Bondoux et al (1991), Jandik et al (1992), Saulnier et al (1992) et Louaileche et al (1993). L'électrolyte est composé de chromate

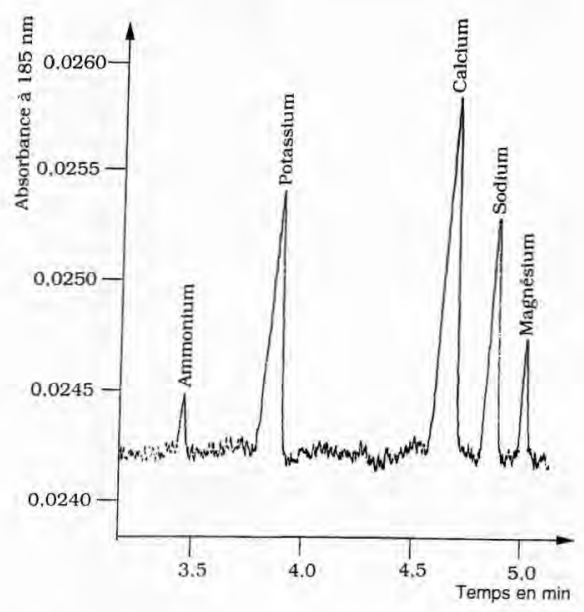

Fig 1. Électrophorégramme type des cations d'un lactosérum acide industriel (technologie pâte molle).

Electrophoregram of cations from industrial acid whey (from moulded curd cheese making). de sodium tétrahydraté (Aldrich, Milwaukee, WI, États-Unis) et d'OFM Anion BT ${ }^{\mathrm{TM}}$ (Waters, Milford, MA, Etats-Unis). Le mode d'injection est le même que celui des cations mais la détection est réalisée à $254 \mathrm{~nm}$ (fig 2 ).

\section{Expression des résultats}

Nous avons calculé la moyenne, l'écart type, le coefficient de variation, les valeurs minimale et maximale pour chacune des deux technologies de lactosérums acides (cinq sérums de pâtes fraîches et sept sérums de pâtes molles). Les résultats sont exprimés en $\%$ de la matière sèche (\%MS). Un test de Student a été réalisé entre ces deux groupes (lactosérums acides de pâtes fraîches et lactosérums acides de pâtes molles).

\section{RÉSULTATS ET DISCUSSION}

\section{Composition en cations}

L'analyse statistique des deux groupes de lactosérums et les données de la littérature sont rapportées dans le tableau I.

Pour le potassium, des différences significatives existent entre les deux groupes de lactosérums. Nos résultats se rapprochent

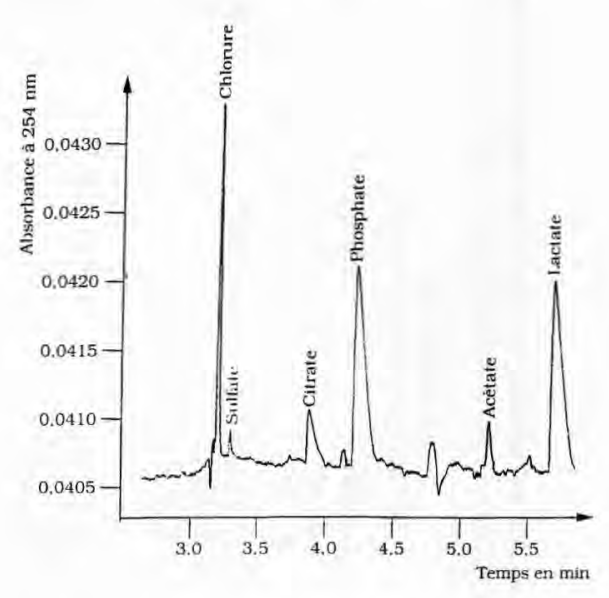

Fig 2. Électrophorégramme type des anions d'un lactosérum acide industriel (technologie pâte molle).

Electrophoregram of anions from industrial acid wheys (from moulded curd cheese making). 
Tableau I. Données statistiques en cations de deux groupes de lactosérums acides exprimées en $\%$ de la matière sèche $(\% \mathrm{MS})$.

Statistical cation values from both groups of industrial acid wheys expressed in $\%$ of dry matter.

\begin{tabular}{|c|c|c|c|c|c|c|}
\hline & \multicolumn{2}{|c|}{$\begin{array}{l}\text { Lactosérums de } \\
\text { technologies pâtes fraîches }\end{array}$} & \multicolumn{2}{|c|}{$\begin{array}{c}\text { Lactosérum de technologies } \\
\text { pâtes molles }\end{array}$} & \multicolumn{2}{|c|}{$\begin{array}{c}\text { Comparaison } \\
\text { des deux technologies }\end{array}$} \\
\hline & $\begin{array}{l}\text { Présente } \\
\text { étude }\end{array}$ & Littérature & $\begin{array}{l}\text { Présente } \\
\text { étude }\end{array}$ & Littérature & $\begin{array}{l}\text { Student } \\
(t)\end{array}$ & $\begin{array}{l}\text { Seuil de } \\
\text { signification }\end{array}$ \\
\hline $\begin{array}{l}\text { Potassium } \\
\text { Moyenne } \pm \text { écart type } \\
\text { Mini / maxi } \\
\text { Coefficient de variation }\end{array}$ & $\begin{array}{c}2,74 \pm 0,29 \\
2,34 / 3,11 \\
10,6 \%\end{array}$ & $2,83^{b}$ & $\begin{array}{c}3,10 \pm 0,19 \\
2,82 / 3,46 \\
6,1 \%\end{array}$ & $2,22^{\mathrm{a}} ; 3,27^{\mathrm{b}} ; 2,47^{\mathrm{c}}$ & 2,34 & 0,05 \\
\hline $\begin{array}{l}\text { Calcium } \\
\text { Moyenne } \pm \text { écart type } \\
\text { Mini / maxi } \\
\text { Coefficient de variation }\end{array}$ & $\begin{array}{c}1,29 \pm 0,28 \\
0,92 / 1,62 \\
21,7 \%\end{array}$ & $1,67^{b} ; 1,90^{d}$ & $\begin{array}{c}2,06 \pm 0,61 \\
1,23 / 3,07 \\
29,6 \%\end{array}$ & $1,43^{\mathrm{a}} ; 0,88^{\mathrm{b}} ; 2,08^{\mathrm{c}}$ & 2,41 & 0,05 \\
\hline $\begin{array}{l}\text { Sodium } \\
\text { Moyenne } \pm \text { écart type } \\
\text { Mini / maxi } \\
\text { Coefficient de variation }\end{array}$ & $\begin{array}{c}1,14 \pm 0,15 \\
0,94 / 1,36 \\
13,2 \%\end{array}$ & $0,98^{d}$ & $\begin{array}{c}1,11 \pm 0,25 \\
0,81 / 1,49 \\
22,5 \%\end{array}$ & $0,66^{\mathrm{a}} ; 0,88^{\mathrm{c}}$ & 0,23 & NS \\
\hline $\begin{array}{l}\text { Magnésium } \\
\text { Moyenne } \pm \text { écart type } \\
\text { Mini / maxi } \\
\text { Coefficient de variation }\end{array}$ & $\begin{array}{c}0,13 \pm 0,01 \\
0,11 / 0,15 \\
7,7 \%\end{array}$ & $0,15 b$ & $\begin{array}{c}0,19 \pm 0,03 \\
0,14 / 0,25 \\
15,8 \%\end{array}$ & $0,15^{\mathrm{a}} ; 0,15^{\mathrm{b}}$ & 3,74 & 0,01 \\
\hline $\begin{array}{l}\text { Ammonium } \\
\text { Moyenne } \pm \text { écart type } \\
\text { Mini / maxi } \\
\text { Coefficient de variation }\end{array}$ & $\begin{array}{c}0,13 \pm 0,12 \\
0 / 0,28 \\
92,3 \%\end{array}$ & 1 & $\begin{array}{c}0,23 \pm 0,11 \\
0,13 / 0,41 \\
47,8 \%\end{array}$ & $0,22^{c}$ & 1,36 & NS \\
\hline
\end{tabular}

NS : non significatif à une probabilité de 0,1

a) Hargove et al, 1976 ; b) Adrian et Bourlier, 1980 ; c) Moulin et Galzy, 1984 ; d) Sottiez, 1985. 
de ceux d'Adrian et Bourlier (1980), qui constatent également une teneur plus importante en potassium dans les lactosérums acides de pâtes molles comparée à celle des lactosérums de pâtes fraîches. En revanche, Hargrove et al (1976) trouvent des valeurs plus faibles que les nôtres dans le cas de la technologie pâte molle.

De significatives variations en calcium existent selon l'origine du lactosérum. En effet, les lactosérums issus de technologies pâtes fraîches ont une moyenne de $1,29 \% \mathrm{MS}$, tandis que ceux issus de technologies pâtes molles ont une moyenne de $2,06 \% \mathrm{MS}$. Cette dernière valeur est en parfait accord avec celle rapportée par Moulin et Galzy (1984), mais est éloignée de celle publiée par Adrian et Bourlier 1980 (0,88 \% MS). Les coefficients de variation importants au sein des deux groupes corroborent des observations antérieures (Saulnier et al, 1995) pour d'autres lactosérums acides industriels.

Les valeurs du sodium obtenues sont proches de celles rapportées par Moulin et Galzy (1984) et Sottiez (1985) pour des technologies identiques. Les moyennes des deux groupes de lactosérums étudiées ne sont pas significativement différentes. Notons que d'importants écarts existent pour les lactosérums de caséines. Sottiez (1985) trouve une teneur en sodium de $2,95 \%$ tandis que Kelly (1991) et Jeantet (1995) trouvent respectivement $0,80 \%$ et $0,64 \%$.

La teneur moyenne en magnésium pour chaque type de lactosérums est en accord avec les valeurs rapportées dans la littérature. Néanmoins, les lactosérums de pâtes fraîches ont un taux moyen légèrement plus faible $(0,13 \% \mathrm{MS})$ que ceux issus de la fabrication de pâtes molles $(0,19 \%$ MS) et les deux groupes sont significativement différents.

La CIA ${ }^{T M}$ permet également de doser l'ion ammonium. La moyenne, en accord avec Moulin et Galzy (1984), est de l'ordre de $0,2 \%$ MS pour la technologie pâte molle.
Quant aux lactosérums de pâtes fraîches, la moyenne n'a aucune signification car deux lactosérums ont été préconcentrés par osmose inverse. En effet, au cours de ce procédé, l'ion ammonium traverse les membranes d'osmose inverse et sa teneur s'en trouve bien entendu réduite dans le lactosérum.

\section{Composition en anions}

L'analyse statistique des deux groupes de lactosérums et les données de la littérature sont rapportées dans le tableau II.

Les valeurs moyennes en chlorure des deux groupes de lactosérums acides industriels ne sont pas significativement différentes. Elles sont en parfait accord avec celles trouvées par Hargrove et al (1976) pour la technologie pâte molle. Sottiez (1985) a rapporté des valeurs plus faibles pour des lactosérums de pâtes fraîches.

Pour les lactosérums étudiés, nous constatons une grande homogénéité dans la composition en sulfate. Nous n'avons relevé aucune donnée bibliographique pour cet ion.

Les moyennes des deux technologies en phosphates sont significativement différentes. En général, les valeurs rapportées dans la littérature expriment la totalité des phosphates (minéral et organique). Afin de pouvoir comparer nos résultats où uniquement le phosphate minéral est dosé par $\mathrm{CIA}^{\mathrm{TM}}$, nous utilisons un facteur correctif de 1,25 calculé selon Alais (1984). Il en résulte que nos résultats sont voisins de ceux de Hargrove et al (1976), de Adrian et Bourlier (1980) et de Moulin et Galzy (1984) pour les technologies pâtes molles.

En revanche, en technologies pâtes fraîches, notre moyenne est très en deçà de celle rapportée dans la littérature. Comme pour le calcium, les teneurs en phosphate sont beaucoup plus faibles pour les lactosérums issus de technologies pâtes fraîches $(1,38 \% \mathrm{MS})$ que pour ceux issus de technologies pâtes molles 
Tableau II. Données statistiques en anions de deux groupes de lactosérums acides exprimée en \% de la matière sèche (\%MS). Statistical anion values from both groups of industrial acid wheys expressed in \% of dry matter.

\begin{tabular}{|c|c|c|c|c|c|c|}
\hline & \multicolumn{2}{|c|}{$\begin{array}{c}\text { Lactosérums de } \\
\text { technologies pâtes fraîches }\end{array}$} & \multicolumn{2}{|c|}{$\begin{array}{l}\text { Lactosérum de technologies } \\
\text { pâtes molles }\end{array}$} & \multicolumn{2}{|c|}{$\begin{array}{c}\text { Comparaison } \\
\text { des deux technologies }\end{array}$} \\
\hline & $\begin{array}{l}\text { Présente } \\
\text { étude }\end{array}$ & Littérature & $\begin{array}{l}\text { Présente } \\
\text { étude }\end{array}$ & Littérature & $\begin{array}{l}\text { Student } \\
\text { (t) }\end{array}$ & $\begin{array}{l}\text { Seuil de } \\
\text { signification }\end{array}$ \\
\hline \multicolumn{7}{|l|}{ Chlorure } \\
\hline Moyenne \pm écart type & $1,99 \pm 0,25$ & $1,51^{d}$ & $2,15 \pm 0,08$ & $2,11^{a}$ & 1,44 & NS \\
\hline Mini / maxi & $1,70 / 2,31$ & & $2,07 / 2,30$ & & & \\
\hline Coefficient de variation & $12,5 \%$ & & $3,7 \%$ & & & \\
\hline \multicolumn{7}{|l|}{ Sulfate } \\
\hline Moyenne \pm écart type & $0,14 \pm 0,04$ & 1 & $0,15 \pm 0,02$ & I & 0,20 & NS \\
\hline Mini / maxi & $0,10 / 0,20$ & & $0,10 / 0,17$ & & & \\
\hline Coefficient de variation & $28,6 \%$ & & $13,3 \%$ & & & \\
\hline \multicolumn{7}{|l|}{ Phosphate } \\
\hline Moyenne \pm écart type & $1,38 \pm 0,19$ & $3,47^{b} ; 3,40^{d}$ & $3,78 \pm 1,50$ & $3,22^{a} ; 3,40^{b} ; 3,32^{c}$ & 3,24 & 0,01 \\
\hline Mini / maxi & $1,14 / 1,65$ & & $1,44 / 5,55$ & & & \\
\hline Coefficient de variation & $13,7 \%$ & & $39,7 \%$ & & & \\
\hline \multicolumn{7}{|l|}{ Citrate } \\
\hline Moyenne \pm écart type & $2,21 \pm 0,33$ & 1 & $2,29 \pm 0,62$ & $0,43^{c}$ & 0,24 & NS \\
\hline Mini / maxi & $1,60 / 2,52$ & & $1,21 / 3,09$ & & & \\
\hline Coefficient de variation & $14,9 \%$ & & $27,1 \%$ & & & \\
\hline \multicolumn{7}{|l|}{ Acétate } \\
\hline Moyenne \pm écart type & $0,16 \pm 0,03$ & 1 & $0,43 \pm 0,26$ & 1 & 2,15 & 0,1 \\
\hline Mini / maxi & $0,12 / 0,19$ & & $0,06 / 0,92$ & & & \\
\hline Coefficient de variation & $18,75 \%$ & & $60 \%$ & & & \\
\hline \multicolumn{7}{|l|}{ Lactate } \\
\hline Moyenne \pm écart type & $1,84 \pm 0,39$ & $10,00^{d}$ & $5,82 \pm 2,48$ & $12,58^{\mathrm{C}}$ & 3,25 & 0,01 \\
\hline Mini / maxi & $1,46 / 2,36$ & & $2,11 / 9,28$ & & & \\
\hline Coefficient de variation & $21,2 \%$ & & $42,6 \%$ & & & \\
\hline
\end{tabular}

NS : non significatif à une probabilité de 0,1

a) Hargove et al, 1976 ; b) Adrian et Bourlier, 1980 ; c) Moulin et Galzy, 1984 ; d) Sottiez, 1985. 
$(3,78 \% \mathrm{MS})$. Cette différence entre les deux technologies n'est pas, à notre connaissance, soulignée dans la littérature. Elle est à imputer à la diversité entre les usines pour une même technologie fromagère dont les lactosérums acides sont issus. La quantité maximale possible en matière minérale d'un sérum est nécessairement dépendante de la richesse en minéraux du lait dont il provient. En effet, le complexe phosphocaséinate est entièrement déminéralisé, le lactosérum doit contenir la quasi-totalité des matières minérales du lait. Ce n'est pas le degré d'acidité finale (exprimé en degré Dornic) atteint par le lait qui représente le facteur déterminant dans la déminéralisation du complexe, mais c'est la durée du maintien en milieu acide du lait ou du caillé (Pien, 1943). Cela peut expliquer les taux élevés de phosphate inorganique et de calcium dans les lactosérums acides de technologies pâtes molles où le temps de contact caillé-lactosérum est plus important que pour les lactosérums issus de technologies pâtes fraîches.

Peu de valeurs concernant les acides organiques acétique, lactique et citrique ont été relevées dans la littérature. Moulin et Galzy (1984) annoncent une teneur en acide citrique de $0,43 \%$ MS (technologies pâtes molles). Mais cet acide peut être métabolisé par les ferments lactiques (Streptoccocus diacetylactis, leuconostocs, etc) utilisés classiquement dans ces technologies. Or, si une mauvaise collecte du lactosérum est faite, les micro-organismes s'y développent et continuent à consommer ce substrat (Saulnier et al, 1995). C'est une des raisons pour lesquelles nous avons congelé les lactosérums dès leur collecte. Toutefois, les moyennes des deux groupes de lactosérums acides étudiés sont significativement différentes pour les acétates et les lactates. Les lactosérums de pâtes fraîches sont caractérisés par des teneurs plus faibles en ces derniers acides organiques comparées à celles des lactosérums de pâtes molles. Les valeurs des coefficients de variations sont élevées pour ces anions dans les sérums issus de technologies pâtes molles.

Par ailleurs, les lactosérums étudiés ne contiennent pas de nitrite et de nitrate (concentration inférieure au seuil de détection de $10 \mathrm{ppb}$ ).

\section{Corrélations entre les différents ions et acides organiques}

Nous avons réalisé une matrice des corrélations des différentes espèces minérales et organiques dosées pour chacun des deux groupes de lactosérums. Pour les technologies pâtes fraîches, la matrice des corrélations est statistiquement inexploitable (résultats non publiés). À l'inverse, en technologies pâtes molles (tableau III), nous remarquons des corrélations significatives $(p=0,05)$ pour les espèces suivantes : calcium, magnésium, phosphate, acétate et lactate.

Nous observons (fig 3) que de forts taux de lactate entraînent des taux plus élevés en calcium et phosphate. Ces résultats ne sont pas surprenants pour des lactosérums acides. En effet, l'acidification du lait ou du caillé par les micro-organismes produisant de l'acide lactique entraîne une solubilisation du phosphate de calcium minéral de la micelle qui se retrouvera donc dans le lactosérum (Brulé et al, 1978 ; Pierre et al, 1983 ; Pouliot et al, 1989 a, b, c ; Saulnier et al, 1992 ; Schmitt et al, 1993 ; Pouliot et Boulet, 1995). La corrélation hautement significative $(p=0,01)$ du phosphate en fonction du calcium $(Y=2,66 X-0,67$, $r=0,87, n=7$ ) le prouve et confirme la forte minéralisation des lactosérums acides de pâtes molles. À pH 5,20 , le phosphate minéral de la micelle se trouve entièrement solubilisé (Le Graet et Brulé, 1993), alors qu'il faut atteindre un $\mathrm{pH}$ de 3,50 pour que la totalité du calcium et du magnésium devienne soluble. 
Tableau III. Matrice des corrélations des anions et cations des lactosérums de pâtes molles. Correlation matrix of anion and cation results from moulded curd wheys.

\begin{tabular}{|c|c|c|c|c|c|c|c|c|c|c|}
\hline & Calcium & Magnésium & Phosphate & Acétate & Lactate & Citrate & Potassium & Sodium & Chlorure & Sulfate \\
\hline Calcium & 1 & & & & & & & & & \\
\hline Magnésium & $0,76^{*}$ & 1 & & & & & & & & \\
\hline Phosphate & $0,87^{\star}$ & 0,62 & 1 & & & & & & & \\
\hline Acétate & $0,95^{\star}$ & 0,64 & $0,90^{*}$ & 1 & & & & & & \\
\hline Lactate & $0,92^{*}$ & 0,62 & $0,96^{*}$ & $0,95^{\star}$ & 1 & & & & & \\
\hline Citrate & $-0,23$ & 0,21 & $-0,29$ & $-0,49$ & $-0,36$ & 1 & & & & \\
\hline Potassium & 0,48 & $-0,04$ & 0,42 & 0,64 & 0,55 & $-0,72^{\star}$ & 1 & & & \\
\hline Sodium & $-0,80^{*}$ & $-0,45$ & $-0,69$ & $-0,65$ & $-0,75^{\star}$ & $-0,01$ & $-0,23$ & 1 & & \\
\hline Chlorure & 0,49 & $0,76^{\star}$ & 0,25 & 0,31 & 0,38 & 0,31 & $-0,16$ & $-0,41$ & 1 & \\
\hline Sulfate & $-0,20$ & $-0,53$ & $-0,03$ & 0,01 & 0,08 & $-0,57$ & 0,64 & 0,25 & $-0,33$ & 1 \\
\hline
\end{tabular}

* Valeur significative à une probabilité de 0,05. 


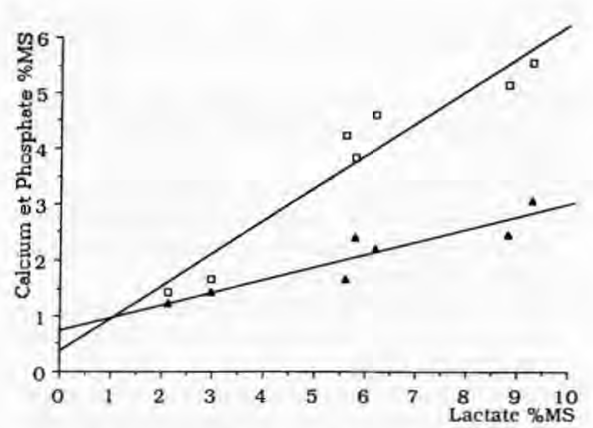

Fig 3. Évolution du taux de calcium $(\boldsymbol{\Delta})$ en fonction du lactate pour les lactosérums acides de technologies pâtes molles $(y=0,225 x+0,75$, $r=0,92, n=7$ ) et du taux de phosphate (a) en fonction du lactate $(y=0,58 x+0,385, r=0,96$, $n=7$ ).

Evolution of calcium values ( $\mathbf{\Delta}$ ) as a function of lactate from industrial acid wheys (moulded curd cheese making) $(y=0,225 x+0,75, r=0,92$, $n=7$ ) and phosphate (a) values as a function of lactate $(y=0,58 x+0,385, r=0,96, n=7)$.

Compte tenu du pouvoir tampon du lactosérum, ce $\mathrm{pH}$ de 3,50 n'est jamais atteint et on ne pourra donc pas avoir dans les lactosérums la totalité du calcium du lait dont il est issu. Une corrélation hautement significative $(p=0,01)$ existe entre le taux d'acétate et celui du lactate $(Y=0,10 X$ $0,13, r=0,95, n=7$ ). Elle est à relier avec l'emploi de souches homo- et hétérofermentaires en fromagerie. Notons qu'une souche homofermentaire qui produit de l'acide lactique en grande quantité peut produire également une plus faible quantité d'acide acétique (Louaileche et al, 1993).

\section{CONCLUSION}

Le présent travail souligne la grande hétérogénéité minérale et organique due aux origines et surtout aux passés technologiques des lactosérums acides, constituant un frein à leur valorisation. En effet, les résultats montrent une grande disparité dans la minéralisation entre les deux technologies (pâtes fraîches et pâtes molles) où des différences significatives existent pour les teneurs en potassium, calcium, magnésium, phosphate, acétate et lactate. De plus, au sein d'une même technologie, d'importants coefficients de variation sont relevés, notamment pour le calcium, le phosphate, l'acétate et le lactate.

Avec l'apparition des nouvelles techniques de déminéralisation, que sont la nanofiltration d'une part et la déionisation en continu (CDI) (Millipore, Bedford, Ma, États-Unis) d'autre part, il est nécessaire d'avoir une parfaite maitrise de la composition organo-minérale des lactosérums (Pontalier et al, 1995). De plus, récemment, Pouliot et Boulet (1995), montrent que le rapport molaire cations/anions influe sur la stabilité thermique des laits concentrés. Mais ce rapport molaire conditionne également la stabilité thermique des lactosérums et de leurs dérivés (Dalan et al, 1972; Gruetzmacher et Bradley, 1991). En effet, plus il augmente, moins les produits sont thermiquement stables. II est donc indispensable d'avoir une méthode rapide pour appréhender la composition organominérale des produits laitiers. La CIA ${ }^{T M}$ possède, selon nous, toutes les caractéristiques pour répondre à cette attente : polyvalence, performances d'analyse, critères économiques.

\section{REMERCIEMENTS}

Ce travail a été réalisé grâce à une aide de la mission recherche de la région Lorraine. Nous remercions également le personnel de la SA Lacto Sérum France pour son aide dans le plan d'échantillonnage et ses précieux conseils.

\section{RÉFÉRENCES}

Adrian J, Bourlier G (1980) Composition minérale du lactosérum. Lait 60, 447-457

Alais C (1981) La valorisation du lactosénum, les bases et les problèmes. Tech Lait 952, 7-10

Alais C (1984) Science du lait. Principes des techniques laitières. $4 \mathrm{e}$ edn, Sepaic, Paris

Blanc B (1969) Molke und daraus hergestellte erzeugnisse, aspekte der zusammensetzung und emah- 
rung des menschen. IDF-Seminar on whey processing and utilization. Weihenstephan, Germany, 1113 November

Bondoux G, Jandik P, Jones WR (1991) Analyse des anions inorganiques et organiques, des cations alcalins, des alcalino-terreux et des métaux par électrophorèse capillaire (CIA ${ }^{\mathrm{TM}}$ : Capillary Ion Analysis). Spectra $2000158,47-51$

Brulé G, Real Del Sol E, Fauquant G, Fiaud C (1978) Mineral salts stability in aqueous phase of milk: influence of heat treatment. J Dairy Sci61, 1225-1232

Dalan E, Groux MJA, Hidalgo J (1972) Procédé de préparation d'une fraction soluble des protéines du petit lait. Brevet $\mathrm{CH} 13.272 / 72$

Gruetzmacher TJ, Bradley RL Jr (1991) Acid whey as a replacement for sodium caseinate in spray-dried coffee whiteners. J Dairy Sci 74, 2838-2849

Hargrove RE, McDonough FE, Lacroix DE, Alford JA (1976) Production and properties of deproteinized whey powder. J Dairy Sci 59, 25-33

Jandik P, Jones WR, Weston A, Brown PR (1992) Electrophoretic capillary ion analysis: Origins, principles, and applications. LC-GC Int 5, 20-27

Jeantet R (1995) Nanofiltration de liquides laitiers. Thèse ENSA, Rennes, France, 139 pp

Kelly J (1991) Partial desalination of whey by nanofiltration. Thèse de l'université de Limerick, Irlande $147 \mathrm{pp}$

Le Graet Y, Brulé G (1993) Les équilibres minéraux du lait : influence du $\mathrm{pH}$ et de la force ionique. Lait 73 , 51-60

Lindeberg $\mathrm{J}$ (1995) Capillary electrophoresis in quantitative food analysis: a survey. In: Current status and future trends. Euro Food Chem VIII, 3, 423-425

Louaileche H, Bracquart P, Saulnier F, Desmazeaud M, Linden G (1993) Carbon dioxide effects on growth and metabolites of morphological variants of Steptococcus thermophilus. J Dairy Sci 76, 3683-3689

Meréo M (1971) Les utilisations industrielles du sérum de fromagerie. Ind Alim Agric 88, 817-823

Moulin G, Galzy P (1984) Whey, a potential substrate for biotechnology. In: Biotechnology genetic engineering reviews, vol I (GE Russell, ed), Intercept, Newcastle upon Tyne, 347-374
Pien J (1943) Utilisation des sérums de fromageries et des lactoprotéines dans l'alimentation. Lait 23, 193222

Pierre A, Brulé G, Fauquant J (1983) Étude de la mobilité du calcium dans le lait à l'aide du calcium ${ }^{45}$. Lait 63 , 473-489

Pontalier PY, Ismail A, Ghoul M (1995) Étude de l'influence des conditions opératoires sur la séparation sélective des ions par des membranes de nanofiltration. Ind Alim Agric 112, 642-646

Pouliot Y, Boulet M (1995) Observations on the seasonal variations in the salt balance of concentrated milk. Int Dairy J 5, 75-85

Pouliot Y, Boulet M, Paquin P (1989a) Observations on the heat induced salt balance changes in milk. Effect of heating time between $40^{\circ} \mathrm{C}$ and $90^{\circ} \mathrm{C}$. J Dairy Res 56, 85-92

Pouliot Y, Boulet M, Paquin P (1989b) Observations on the heat induced salt balance changes in milk. II. Reversibility on cooling. J Dairy Res 56, 193-199

Pouliot Y, Boulet M, Paquin P (1989c) An experimental technique for the study of milk salt balance. J Dairy Sci $72,36-40$

Rolet A (1920) Les industries annexes de la laiterie, utilisation des sous-produits et résidus. Baillière, Paris, 216

Saulnier F, Schmitt M, Linden G (1992) Monitoring of temperature induced shifts of ionic equilibria in milk. International Ion Chromatography Symposium. Linz, Autriche, 21-24 September

Saulnier F, Ferrero F, Bottero JY, Linden G (1995) Variations de composition et nature de la fraction insoluble des lactosérums industriels. Lait 75, 93-100

Schmitt M, Saulnier F, Malhautier L, Linden G (1993) Effect of temperature on the salt balance of milk studied by capillary ion electrophoresis. J Chromatogr $640,419-424$

Sottiez P (1985) Produits dérivés des fabrications fromagères. In: Laits et produits laitiers, vol II (FM Luquet, ed), Tec et Doc Lavoisier, Paris, 357-392

Veisseyre R (1979) Technologie du lait, $3^{e}$ edn, La Maison Rustique, Paris

Webb BH (1972) Recycling whey for profitable uses. Am Dairy Rev 34, 32A-32D 